\title{
Effect of capping agents on optical and antibacterial properties of cadmium selenide quantum dots
}

\author{
DEEPIKA $^{1}$, RAKESH DHAR ${ }^{1, *}$, SUMAN SINGH $^{2}$ and ATUL KUMAR ${ }^{1}$ \\ ${ }^{1}$ Department of Applied Physics, Guru Jambheshwar University of Science and Technology, Hisar 125 001, India \\ ${ }^{2}$ Central Scientific Instruments Organization (CSIR-CSIO), Chandigarh 160 030, India
}

MS received 12 August 2014; accepted 16 April 2015

\begin{abstract}
Cadmium selenide quantum dots (CdSe QDs) were synthesized in aqueous phase by the freezing temperature injection technique using different capping agents (viz. thioglycolic acid, 1-thioglycerol, L-cysteine). Absorption spectra of CdSe QDs exhibited a blue shift as compared to its bulk counterpart, which is an indication of quantum confinement effect. The photoluminescence spectra of CdSe QDs confirmed that the particles are poly-dispersed and possess enhanced luminescent property, depending upon the chemical nature of capping agents. The QDs have been characterized by Fourier-transform infrared spectroscopy, atomic absorption spectroscopy and transmission electron microscopy. Further, antimicrobial activity of as-prepared QDs has also been investigated using the disk diffusion method.
\end{abstract}

Keywords. Capping agents; quantum confinement; poly-dispersed; luminescent.

\section{Introduction}

Over the past few decades, there has been significant development in the fields of semiconductor nanoparticles (NPs) for various applications. Quantum dots (QDs) provide advantages over organic fluorophores, including good photostability, broad absorption spectra and tunable emission spectra. Among various semiconductor materials, cadmium selenide ( $\mathrm{CdSe}$ ) is an important direct-band semiconductor with bandgap $\left(E_{\mathrm{g}}\right)$ of $1.74 \mathrm{eV}$, having unique optical properties resulting from quantum confinement effect and wide tuning of bandgap with particle size. ${ }^{1-3}$ Main interest in studying the CdSe QDs is related to their preparation and optical properties, which make them suitable in application such as biomedical labelling, ${ }^{4}$ solar energy conversion ${ }^{5}$ and photoelectronics. ${ }^{6}$ Numerous methods have been developed for the preparation of CdSe QDs and can be summarized mainly into two chemical routes, one is non-aqueous trioctyl phosphine/trioctyl phosphine oxide (TOP/TOPO) route and another is aqueous route that employs use of different thiols, thioacids and amides as stabilizing agent. Aqueous phase synthesis of QDs may be an excellent alternative to an organic phase fabrication. Aqueous phase synthesis can be very simple and is highly reproducible, as well as relatively economical and environment friendly and resulting QDs are more stable, water soluble and biocompatible. ${ }^{7-9}$ The kind of stabilizer used to passivate QDs is of great importance, as it could lead to different surface

\footnotetext{
*Author for correspondence (rakesh285@gmail.com)
}

structures and affect the nucleation and growth kinetics of QDs. ${ }^{10}$ For the preparation of QDs, organic molecules (viz., thioglycolic acid (TGA), 1-thioglycerol (TGH), L-cystene (L-cys)) with both sulphydryl and carboxyl functional groups have been widely adopted as capping molecules. The sulphydryl group can coordinate to the NPs, whereas the carboxyl group can contribute to the electrostatic stabilization of the colloidal NPs as well as to their further surface modification for various applications. ${ }^{11}$

Due to their inherent chemical composition (heavy metals, $\mathrm{Cd}, \mathrm{Te}, \mathrm{Se}$, etc.) or as a consequence of their nanoscale properties, research concerning the potential toxicity of QDs has also gained a great amount of interest. The evaluation of QD toxicity to bacteria is complex as it is a function of: (1) QD core material and size, (2) QD coating, (3) bacterial strain and (4) conditions in which they are used. ${ }^{12}$ In the present study, antimicrobial activity of a series of QDs capped with different capping ligands, such as TGA, L-cys, TGH, has been studied using Staphylococus aureus as a bacterial strain. The results demonstrated that surface modification due to different capping ligands plays important role in the antibacterial activity of QDs.

\section{Experimental}

\subsection{Materials}

Cadmium acetate dehydrate $\left(\mathrm{CdCl}_{2} \cdot 2 \mathrm{H}_{2} \mathrm{O}\right)$, TGA, sodium borohydride $\left(\mathrm{NaBH}_{4}\right)$, TGH, L-cys, Luria broth and agar were from Hi Media. Selenium powder was procured from Sigma Aldrich. HPLC grade water with resistivity of 
$18 \mathrm{M} \Omega \mathrm{cm}^{-1}$ was used for the preparation of aqueous solutions.

\subsection{Material characterization}

UV-visible absorption spectra were recorded on a Varian UV-vis spectrophotometer (Cary 5000). Fluorescence emission spectra were collected on Varian fluorescence spectrophotometer (Cary Eclipse), where an excitation wavelength of $400 \mathrm{~nm}$ was used for all the measurements reported here. All optical measurements were carried out at room temperature under ambient conditions. The morphology and size of QDs were determined using transmission electron microscope (TEM; Hitachi, Model no. 7500). For TEM studies, the preparation of sample consisted of dispersing QDs in chloroform and a drop of the dispersion was placed on carbon-coated TEM grids. The solvent evaporated thus leaving a thin layer of material on the grid. The IR spectrum was recorded with a Perkin Elmer spectrum (BX II) to obtain information about the surface of the QDs and the binding of thiol and amide groups with CdSe QDs. For this, the powder samples were mixed with anhydrous potassium bromide $(\mathrm{KBr})$, pelletized, and used for FTIR analysis. AAS was recorded using GBC $932+$ to obtain concentration of cadmium in samples.

\subsection{Synthesis of CdSe QDs}

For the synthesis of QDs, an aqueous solution of cadmium dihydrate and TGA $\left(\mathrm{HSCH}_{2} \mathrm{COOH}\right)$ was prepared in the $1: 4$ and $\mathrm{pH}$ of the solution was adjusted to 12 by adding $1 \mathrm{M}$ solution of $\mathrm{NaOH}$. The solution was deaerated using nitrogen gas $\left(\mathrm{N}_{2}\right)$ bubbling for $30 \mathrm{~min}$. NaHSe solution was prepared by adding sodium borohydride and Se powder in $4 \mathrm{ml}$ distilled water in the ratio $4: 1$. The reacting system was cooled to $0^{\circ} \mathrm{C}$ in dark. During the reaction, a small outlet connected to the flask was kept open to discharge the pressure from the resulting hydrogen gas. The addition of NaHSe through syringe under vigorous magnetic stirring produced bright-yellow, transparent TGA-capped CdSe QDs. QDs were stored at $4{ }^{\circ} \mathrm{C}$ to prevent agglomeration and no precipitates were observed after 30 days. The same procedure was followed to prepare $\mathrm{TGH}$-capped $\mathrm{CdSe}$ and L-cys-capped CdSe. The end products of TGH-capped CdSe and L-cys-capped $\mathrm{CdSe}$ were greenish and greenish yellow, respectively. In all the cases, the QD solutions were precipitated using ethanol and centrifuged 4-5 times to remove excess impurities, which might be present during preparation of QDs.

\subsection{Antibacterial study of CdSe QDs}

The disk diffusion method has been used to evaluate the antimicrobial activity of QDs capped with different capping agents against $S$. aureus (ATCC 6538). The method was performed in Luria broth (LB) medium and solid agar in Petri dish.

\subsection{Preparation of culture plates}

For media preparation, $5 \mathrm{~g}$ Luria broth media and $5 \mathrm{~g}$ agar was taken into $500 \mathrm{ml}$ conical flask containing $250 \mathrm{ml}$ of double distilled water. Solution was mixed thoroughly and kept for autoclaving. Immediately after autoclaving, the solution was placed in water bath at $45-50^{\circ} \mathrm{C}$. This freshly prepared medium was poured into flat-bottomed Petri dish on a level, horizontal surface to give a uniform depth of approximately $4 \mathrm{~mm}$. This corresponds to $60-70 \mathrm{ml}$ of medium for plate with diameters of $150 \mathrm{~mm}$ and 25-30 $\mathrm{ml}$ for plate with a diameter of $100 \mathrm{~mm}$. The agar medium was allowed to cool at room temperature.

\subsection{Inoculation of culture plates}

A sterile cotton swab was dipped into freshly prepared $S$. aureus suspension. The swab was rotated several times and pressed firmly on the wall of the tube above the fluid level to remove excess inoculum from the swab. The dried surface of the culture plate was inoculated by streaking the swab over the entire sterile plate surface. This procedure was repeated by streaking two more times, rotating the plate approximately $60^{\circ}$ each time to ensure an even distribution of inoculum. Finally, the rim of the agar was also swabbed. The lid was left ajar for $5 \mathrm{~min}$, to allow for any excess surface moisture to be absorbed before applying the QDs impregnated filter discs. Whatman filter paper no. 1 was used to prepare discs of approximately $6 \mathrm{~mm}$ in diameter. The discs were placed in a Petri dish and sterilized in a hot air oven. After cooling the discs at room temperature, $10 \mu \mathrm{l}$ of QDs solution (concentration $5 \mathrm{mg} \mathrm{ml}^{-1}$ ) was pipetted on Whatman filter paper dics placed on culture plate. The agar plate containing NPs impregnated filter discs was then incubated for $18 \mathrm{~h}$ at $37^{\circ} \mathrm{C}$ to examine the antimicrobial effect of NPs for which inhibition zone was monitored and measured in millimetre. QD1 represents QDs capped with L-cys, QD2 represents QDs capped with TGA and QD3 represents that with 1-thioglycerol.

To determine cadmium concentration in the solution we performed AAS experiment. Concentration of cadmium was found to be 17.5, 22.5 and $175 \mathrm{ppm}$ in the QDs capped with L-cys, THG and TGA, respectively, in QDs $\left(5 \mathrm{mg} \mathrm{ml}^{-1}\right)$.

\section{Results and discussion}

\subsection{Physical characterization of $Q D s$}

CdSe QDs were characterized for their physical characteristics, which included morphology/shape, structure and 

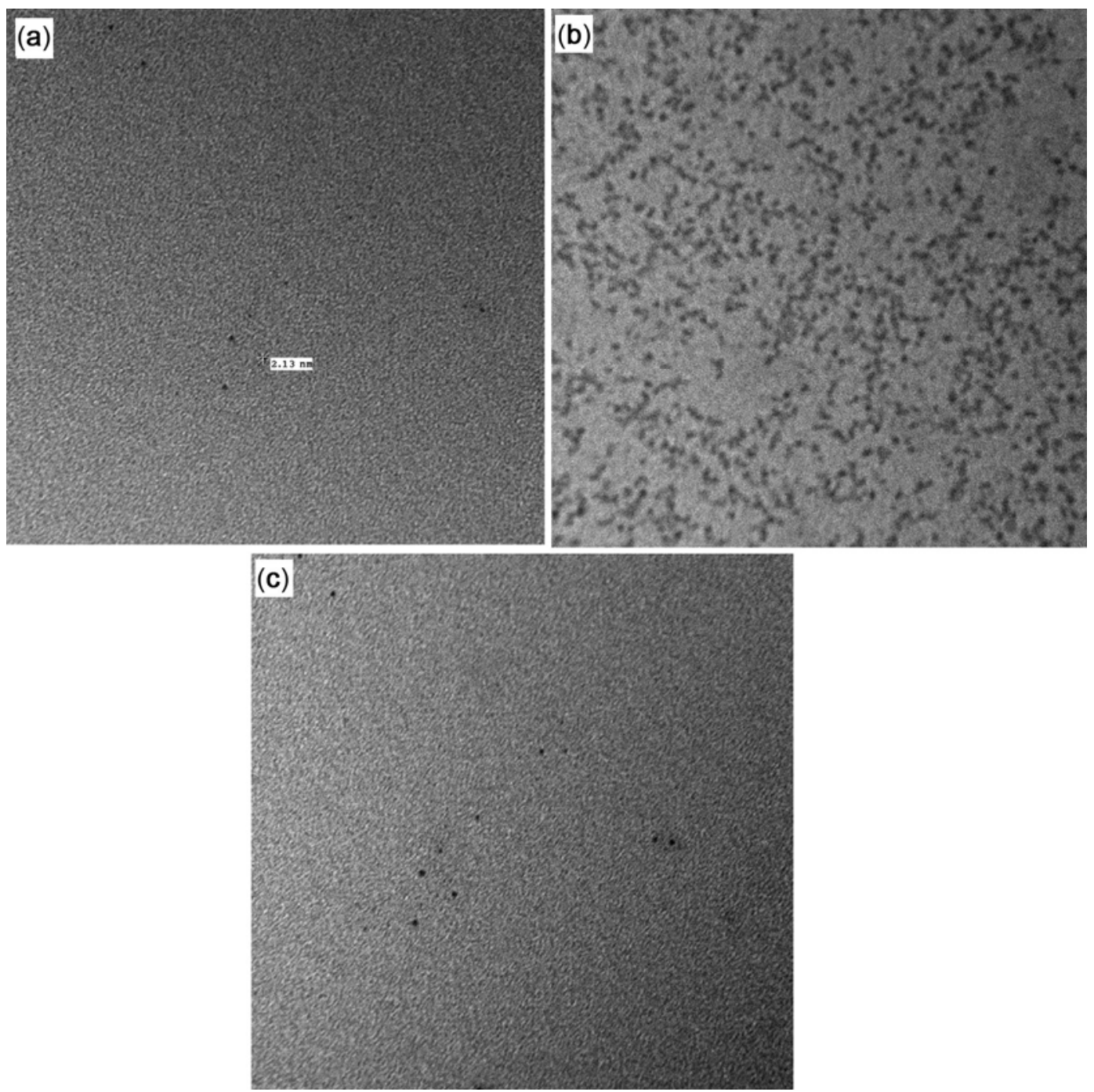

Figure 1. TEM images of CdSe QDs: capped with (a) L-cysteine, (b) thioglycolic acid and (c) 1-thioglycerol.

Table 1. Characteristic absorption bands for different capping agents.

\begin{tabular}{lcl}
\hline Capping agent & $\begin{array}{c}\text { Wavenumber } \\
\left(\mathrm{cm}^{-1}\right)\end{array}$ & \multicolumn{1}{c}{ Band } \\
\hline 1-thioglycerol & 3444 & $\mathrm{O}-\mathrm{H}$ vibration \\
& 2914 & $\mathrm{C}-\mathrm{H}$ stretching \\
& $\begin{array}{c}358.79 \\
\mathrm{C}=\mathrm{O} \text { vibration }\end{array}$ \\
& 705.9 & $\mathrm{C}-\mathrm{S}$ stretching \\
Thioglycolic acid & 3400 & $\mathrm{O}-\mathrm{H}$ vibration \\
& 1349.15 & $\mathrm{C}=\mathrm{O}$ vibration \\
& 1032.3 & $\mathrm{C}-\mathrm{O}$ vibration \\
& 715.25 & $\mathrm{C}-\mathrm{S}$ stretching \\
& 3500 & $\mathrm{O}-\mathrm{H}$ vibration \\
& 2933 & $\mathrm{C}-\mathrm{H}$ stretching \\
& 1651 & $\mathrm{~N}-\mathrm{H}$ bend primary amine \\
& 1384 & $\mathrm{C}=\mathrm{O}$ vibration \\
& 1020 & $\mathrm{C}-\mathrm{N}$ stretching \\
\hline
\end{tabular}

FTIR. Figure 1a-c shows TEM images of L-cys-, TGAand 1-thioglycerol-capped CdSe QDs. TEM images show the presence of dispersed QDs having average size of 2-3 nm, with a 1-2 nm-thick layer of capping agents. The QDs are arranged in regular manner, nearly spherical in size. The sizes mentioned are just an approximation as there can be some factors like background noise that might introduce uncertainty in the measurement of the diameter of NPs.

Figure 2 shows FTIR spectra of CdSe QDs prepared with respective capping agents. A broad absorption peak at around $3400 \mathrm{~cm}^{-1}$ can be assigned to the $\mathrm{O}-\mathrm{H}$ vibration, and strong bands at $2923 \mathrm{~cm}^{-1}$ is due to $\mathrm{C}-\mathrm{H}$ stretching vibrations of the alkyl chains of ligand molecules in all spectra. The absence of the $\mathrm{S}-\mathrm{H}$ stretching mode around $2560 \mathrm{~cm}^{-1}$ in these spectra clearly indicates that thiol group of ligands are bound to surface atoms of QDs through the $\mathrm{Cd}-\mathrm{S}$ bond. It is interesting that all of the 


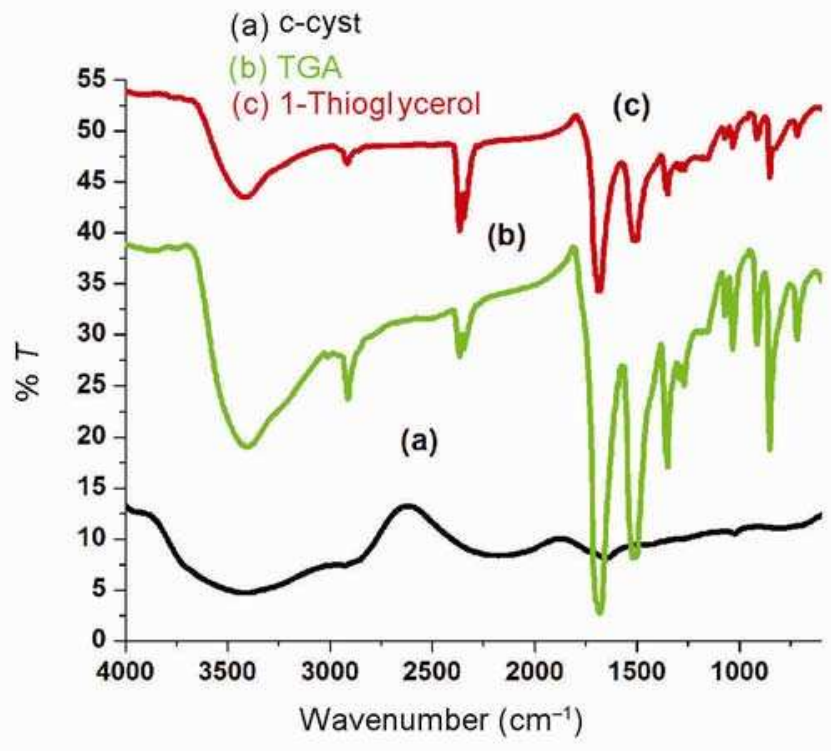

Figure 2. FTIR spectra of QDs: (a) capped with L-cysteine, (b) thioglycolic acid and (c) 1-thioglycerol.

particles show a sharp, prominent peak due to carboxylate anions ( $\mathrm{C}=\mathrm{O}$ vibrations $)$, and this imparts a net negative charge on the outer surface of NPs, which essentially helps QDs not to coagulate and agglomerate. FTIR results are in good agreement with those obtained by other research groups. ${ }^{13}$

\subsection{Optical characterization}

Figure $3 \mathrm{a}$ and $\mathrm{b}$ shows UV-visible and emission spectra of CdSe QDs capped with different capping agents. The UV-visible spectra show absorption peak at $432 \mathrm{~nm}$ for TGA-capped CdSe QDs, at $347 \mathrm{~nm}$ for 1-thioglycerol-capped QDs and at $420 \mathrm{~nm}$ for L-cyscapped QDs. Absorption spectrum of L-cys-capped QDs exhibits a very sharp first peak at $420 \mathrm{~nm}$, together with smaller peaks at 390 and $362 \mathrm{~nm}$. Very sharp absorption peak suggests that the prepared QDs are highly stable as the sharpness and position of the peak are very similar to those for the ultra stable $(\mathrm{CdSe})_{33}$ and $(\mathrm{CdSe})_{34}$ magic clusters grown selectively in the organic phase. ${ }^{14}$ The synthesized CdSe QDs showed a clear shift of the band edge in the colloidal particles $(480 \mathrm{~nm}$ for TGA-capped QDs, $450 \mathrm{~nm}$ for 1-thioglycerol and L-cys) from bulk CdSe $(712 \mathrm{~nm})$ due to quantum confinement effects inside NPs. This blue shift indicates that there is an increase in the bandgap.

The energy bandgap of QDs shown in table 2 was calculated from the Tau plot (figure $3 \mathrm{c}$ ) and using absorption peak. The value of bandgap suggests that these QDs can be used in visible transmitting thin film applications, as the range of bandgap for visible transmitting film
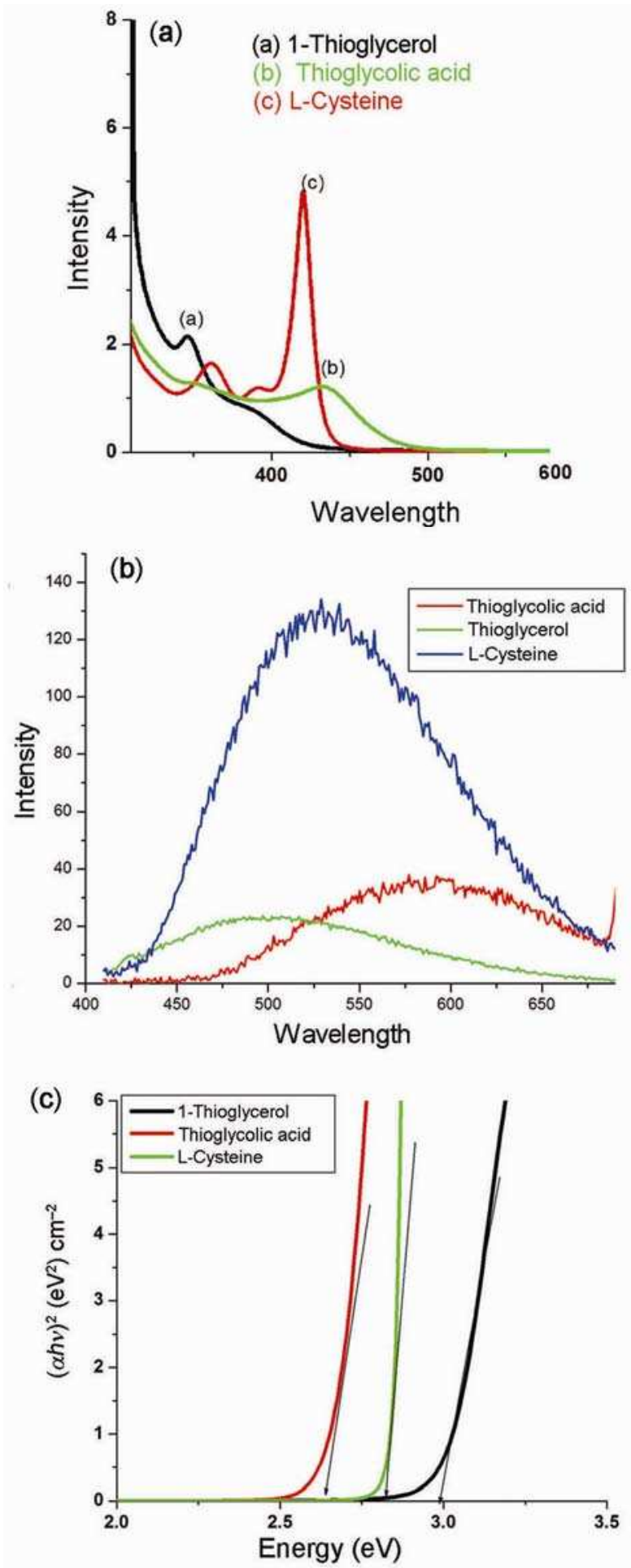

Figure 3. UV-vis absorption spectra of (a) CdSe QDs capped with different capping agents, (b) emission spectra CdSe QDs capped with different capping agents and (c) Tau plot for calculating bandgap of QDs.

is $1.5-3.0 \mathrm{eV} .{ }^{15}$ The particle size has been calculated using Brus's equation (1) ${ }^{16}$ and is shown in table 2.

$$
\begin{aligned}
E_{\mathrm{g}}= & E_{\mathrm{bulk}}+h^{2} / 8 m_{\mathrm{o}} R^{2}\left(1 / m_{\mathrm{e}}^{*}+1 / m_{\mathrm{h}}^{*}\right) \\
& -1.786 e^{2} / 4 \pi \varepsilon_{0} \varepsilon_{\mathrm{r}} R .
\end{aligned}
$$


Table 2. Particle size of QDs, calculated using effective mass approximation and TEM and bandgap calculated from the Tau plot and absorption spectra for different capping agents.

\begin{tabular}{lccccc}
\hline $\begin{array}{l}\text { Capping } \\
\text { agent }\end{array}$ & $\begin{array}{c}\text { Absorption } \\
\text { peak (nm) }\end{array}$ & $\begin{array}{c}\text { Band gap (eV) } \\
\text { using Tau plot }\end{array}$ & $\begin{array}{c}\text { Band gap (eV) } \\
\text { using absorption peak }\end{array}$ & $\begin{array}{c}\text { Size calculated using } \\
\text { Brus equation (nm) }\end{array}$ & $\begin{array}{c}\text { Size calculated } \\
\text { using TEM (nm) }\end{array}$ \\
\hline 1-thioglycerol & 347 & 3.0 & 3.5 & 2.86 & 2 \\
$\begin{array}{l}\text { L-cysteine } \\
\text { Thioglycolic acid }\end{array}$ & 420 & 2.82 & 2.94 & 3.5 & 2.13 \\
\hline
\end{tabular}

It is important to note that these values are estimated using the first excitonic peak in the optical absorption spectra, which always occurs at higher energy than the optical absorption edge. Hence, this estimation of size is always smaller than the size taken from the optical absorption edge. The discrepancies among the sizes estimated from TEM and absorption studies could be attributed to differences in the number of particles involved in the statistics. The size from the TEM image is relative to statistics done on a sample of approximately 100-200 NPs, while number of particles involved in the statistics for absorption is extremely large, which results in a focusing of size distribution. The quality of capping on CdSe QDs was studied using fluorescence spectroscopy. Photoluminescence (PL) spectra of CdSe QDs showed an emission peak at 500, 530 and $590 \mathrm{~nm}$ for TGH-, L-cysand TGA-capped QDs, respectively (figure 3b). The peak are red shifted from that of their absorption wavelength, i.e., 347, 420, $432 \mathrm{~nm}$. The difference between absorption and emission wavelength is known as Stokes shift, which can be explained on the basis of the Frank Condon Principle. The PL intensities of the QDs show the following increasing order: $\mathrm{CdSe}-\mathrm{TGH}<\mathrm{CdSe}-$ TGA $<\mathrm{CdSe}-\mathrm{L}-\mathrm{cys}$ and their FWHM also increased in the same pattern. This indicates that these ligands bind too strongly to the surface of the QDs, avoiding the sharp separation of nucleation and growth under the reaction conditions employed here. High emission intensity of L-cys-capped CdSe QDs can be attributed to the formation of magic clusters. ${ }^{11}$ The energy shift in PL has shown good correlation with the results obtained from other techniques such as UV-visible absorption spectroscopy.

\subsection{Antibacterial study}

QDs capped with L-cys $\left(\mathrm{QD}_{1}\right)$ showed minimum zone of inhibition indicating less antimicrobial activity and TGAcapped CdSe QDs $\left(\mathrm{QD}_{2}\right)$ showed maximum zone of inhibition, indicating more antibacterial activity. TGHcapped QDs $\left(\mathrm{QD}_{3}\right)$ exhibited antibacterial activity in between the two. For antibacterial behaviour, the surface modification is an important factor. In the present study we tried to explain the antibacterial behaviour on the basis of chemical structure of stabilizers used (figure 4). (a)<smiles>NCC(=O)O</smiles>

(b)

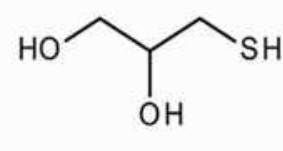

(c)

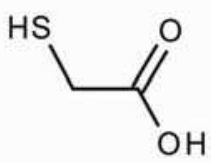

Chemical structure of (a) L-cysteine, (b) 1-thioglycerol and (c) thioglycolic acid.

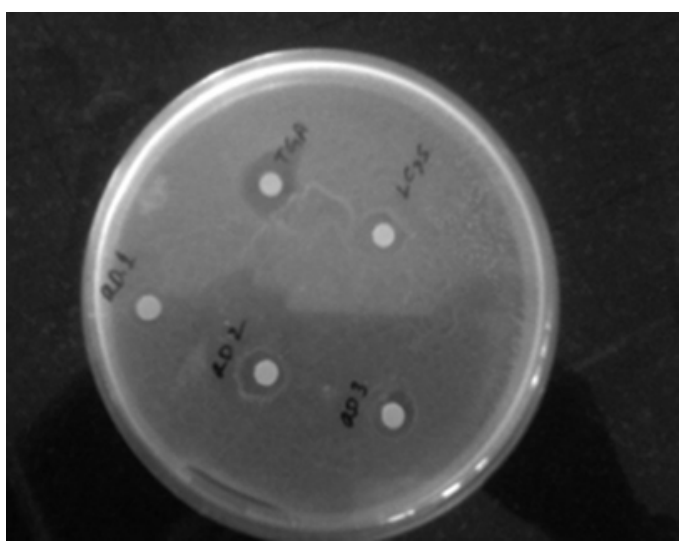

Figure 4. Antibacterial behaviour of L-cys, TGA and TGH and are labelled at $\mathrm{QD}_{1}, \mathrm{QD}_{2}$ and $\mathrm{QD}_{3}$, respectively.

L-cys is $\alpha$-amino acid having three functional groups (amine, sulphydryl and carboxyl). Each of them has lone pair electrons and thus can bear an electric charge, depending on solution $\mathrm{pH}$. It is well known that $-\mathrm{NH}_{2}$ and $-\mathrm{COO}^{-}$can act as a pair of binding sites for metal ions. Primary coordination is in between the sulphydryl group of cys and surface $\mathrm{Cd}$ of the QDs and is expected also because of strong nucleophilicity of the thiolate group (figure 5). At $\mathrm{pH} \sim 12$, amine group of cys bears no electric charge in the solution, and nucleophilicity of a neutral amine group is slightly stronger than that of a carboxylate group. Thus, the amine group may have priority over the carboxylate group for secondary coordination. The carboxyl group exists as a negatively charged carboxylate form, and that contributes to the electrostatic stabilization of the colloidal CdSe QDs. Once the small CdSe QDs are formed in the reaction system, the QDs have a chance of combining with each other through the chelate function between the amino acid and $\mathrm{Cd}_{2}^{+}$. This leads to better surface passivation and as a result, there 


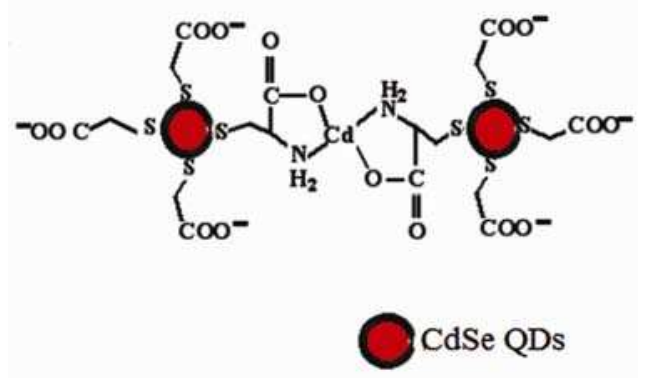

Figure 5. Schematic presentation of binding of Cd with $-\mathrm{NH}_{2}$ group and oxygen of carboxyl group of L-cysteine.

are less chances of leakage of free $\mathrm{Cd}_{2}^{+}$ions in these QDs and hence they show minimum antibacterial activity. ${ }^{17-19}$ Whereas, TGA and TGH both are small ligand groups and the secondary coordination exists between carbonyl oxygen of free acid and $\mathrm{Cd}$ atom at the CdSe particle surface, which provides stability to the prepared QDs but not to the extent of L-cys ligand. As a result, the chances of leakage of free $\mathrm{Cd}_{2}^{+}$ions is maximum in the QDs capped with these ligands ${ }^{20}$ and they show maximum antibacterial activity.

These results are in good agreement with those obtained by other groups ${ }^{21,22}$ and confirm that QDs capped with small ligands like TGA are most toxic to bacteria. At the same time, the formation of reactive oxygen species (ROS) cannot be ignored completely. QDs have bright PL, narrow emission and broad absorption spectra, they can act as photosensitizes; they absorb visible light and generate ROS, resulting in antibacterial activity. ROS are extremely potent, causes serious cell damage by oxidizing the biopolymers like protein and lipids that triggers the cellular signals for programmed cell death, leading to the biocidal effects. ${ }^{23,24}$ On the basis of these, we propose the mechanism of the antibacterial activity of CdSe QDs that involves both the surface modification by capping ligands and a ROS-dependent pathway.

\section{Conclusion}

CdSe QDs capped with different capping agents have been synthesized using a freezing temperature injection technique, resulting in the formation of well-dispersed QDs having a size in the range $2-4 \mathrm{~nm}$. It is clearly shown that the nature of capping agents affects the optical properties of QDs, 1-thioglycerol was the most effective in achieving the lowest size and highest bandgap compared to the other capping agents. L-cys-capped QDs show maximum PL intensity. Antimicrobial behaviour of QDs were also studied and found that QDs capped with L-cys showed minimum antimicrobial activity and that with TGA showed maximum antimicrobial activity. We explained the antimicrobial behaviour of the
QDs on the basis of structure of different stabilizing agents used and L-cys being a bidentate ligand binds effectively with the QDs and minimizes the release of $\mathrm{Cd}$ ions and hence show minimum antimicrobial activity. The future scope of the study lies in tagging these QDs with biomolecules and to use them as biosensors, fluorescent probes, etc.

\section{Acknowledgements}

Deepika is thankful to GJUS\&T and Haryana Board of Technical Education for providing Sir C.V. Raman fellowship. We are also thankful to CIL, GJUS\&T for providing characterization facility.

\section{References}

1. Revaprasadu N and Mlondo S N 2006 Pure Appl. Chem. 78 1691

2. Bera D, Qian L, Tseng T K and Holloway P H 2010 Materials 32260

3. Rajh T, Micic O I and Nozik A J 1993 J. Phys. Chem. 97 11999

4. Drbohlavova J, Adam V, Kizek R and Hubalek J 2009 Int. J. Mol. Sci. 10656

5. Kamat P V 2008 J. Phys. Chem. C 11218737

6. Stokes E B, Stiff Roberts A D and Dameron C T 2006 Electrochem. Soc. Interface 1523

7. Liu L, Peng Q and Li Y 2008 Inorg. Chem. 475022

8. Rogach A L et al 2007 J. Phys. Chem. C 11114628

9. Li Z, Dong C, Tang L, Zhu X, Chen H and Ren J 2011 Luminscence 26439

10. Sobhana S S L, Devi M V, Sastry T P and Manda A B 2011 J. Nanopart. Res. 131747

11. Park Y S et al 2010 ACS Nano 4121

12. Park S, Chibli H, Wong J and Nadeau J L 2011 Nanotechnology 22185101

13. Kalasad M N, Rabinal M K and Mulimani B G 2009 Langmuir 2512729

14. Kasuya A et al 2004 Nat. Mater. 399

15. Cody C A, Brook B G and Abele B 1982 Sol. Energy Mater. 8231

16. Brus L 1986 J. Phys. Chem. 902555

17. Zhang H, Zhou Z, Yang B and Gao M 2003 J. Phys. Chem. B 1078

18. Li J et al 2003 Chem. Commun. 1740

19. Lu Z, Li C M and Bao H 2008 Langmuir 245445

20. Dance I G, Scudder M L and Secomb R 1983 Inorg. Chem. 221794

21. Voura E B, Jaiswal J K, Matoussi H and Simon S M 2004 Nat. Med. 10993

22. Jaiswal J K, Mattoussi H, Mauro J M and Simon S M 2003 Nat. Biotechnol. 2147

23. Schneider R, Wolpert C, Guilloteau H, Balan L, Lambert J and Merlin C 2009 Nanotechnol. 20225101

24. Joshi P et al 2009 J. Nanosci. Nanotechnol. 96427 